\title{
Developing odontoma arising from Calcifying odontogenic cyst: a
} case report

\author{
Monir Moradzadeh Khiavi $^{1}$, Nazanin Mahdavi ${ }^{2}$, and ASOMA AWUDU² \\ ${ }^{1}$ Tehran University of Medical Sciences Dental School \\ ${ }^{2}$ Tehran University of Medical Sciences
}

July 27, 2021

\begin{abstract}
Developing odontoma is a rare mixed odontogenic tumor that can arise with other odontogenic lesions. The association of COC with ameloblastic fibro-odontoma is extremely rare. We report an extremely rare case of developing odontoma arising from a calcifying odontogenic cyst in a 17-year old girl.
\end{abstract}

\section{Introduction}

Developing odontoma replaced ameloblastic fibro-odontoma (AFO) as a new entity in the latest (4th) edition, 2017, of the World Health Organization (WHO) Head and Neck Tumors classification. ${ }^{1}$ The world health organization has classified developing odontoma as a mixed odontogenic tumor. ${ }^{2}$ It is a rare mixed odontogenic tumor characterized by the proliferation of odontogenic epithelium as cords and small islands in a background of primitive ectomesenchymal cells similar to dental papilla and in association with calcified tooth structures including enamel and dentin. ${ }^{3}$

The calcifying odontogenic cyst (COC) is a rare benign odontogenic lesion that exhibits both cystic and neoplastic features. It has a highly diverse histopathologic characteristics. ${ }^{4}$ In 1962 Gorlin described the first case of $\mathrm{COC}$ as a separate entity thus the frequent use of the term Gorlin cyst for this lesion. ${ }^{5}$ COC has a wide variety of clinical and histopathological manifestations and debate about the true nature of this lesion is still ongoing. ${ }^{4,6}$ Following several classifications and reclassifications, the WHO in 2005 recommended and classified it as calcifying cystic odontogenic tumor (CCOT) ${ }^{6}$ In the 2017 classification, the cystic form of the lesion was classified as a developmental cyst whereas the solid form was classified as a mixed odontogenic tumor. $^{7}$

The combination of COC and Ameloblastic fibro-odontoma has been reported before. However this combination is extremely rare. This paper reports the first case of developing odontoma as a new entity arising from calcifying odontogenic cyst.

\section{Case report}

A 17 year-old female was referred to the Oral and Maxillofacial Pathology Department, Tehran University of Medical Sciences, Tehran, Iran, for consultation and diagnosis of a lesion on the right side of her mandible. The previous histopathologic diagnosis of the lesion was ameloblastoma. Before the incisional biopsy, the patient's chief complaint was a swelling in the right posterior mandible for 2 weeks. There was no facial asymmetry. In the intraoral examination, a slight swelling was observed in the right retromolar area. The swelling was non-tender and was firm in consistency. Panoramic and CBCT radiographs showed a welldefined radiolucent lesion that contained areas of radiopacity around the crown of an unerupted third molar. The margins of the lesion were sclerotic (Figure 1) 
In the review of the microscopic slides, a cystic lesion lined by an odontogenic epithelium which consists of cuboidal to columnar basal cells with hyperchromatic nuclei demonstrating reverse polarity was observed. The superficial epithelial cells were loosely arranged and resembled the stellate reticulum of enamel organ. Aggregations of ghost cells were notable within the epithelium (figure 2A). Sections of numerous tooth-germ structures consisting of nests and islands of enamel organ and dental papilla were also evident in the cyst wall. In some areas, tooth buds appeared to originate from the cystic epithelium (figure 2B). There were also sections of developed tooth structures consists of mature enamel cap and tubular dentine (figure 3). Area of ectomesenchyme-like cell proliferation similar to dental papilla were also seen. Based on these histopathologic findings the diagnosis was developing odontoma arising from a calcifying odontogenic cyst. The lesion was treated by conservative excision and there was no recurrence after one-year follow-up (figure 4).

\section{Discussion}

Unlike solitary tumors, hybrid tumors are rare. However, $25 \%$ of cases of calcifying odontogenic cyst (COC) occurs concurrently with other odontogenic lesions and the most common concurrent lesion seen in this association is odontoma. ${ }^{6}$ There are very few cases of ameloblastic fibro-odontoma (AFO) associated with COC and the first reported case was published in $1987 .{ }^{8}$

Developing odontoma was formerly called AFO until 2017 when WHO changed its name. ${ }^{1}$ This report is the first case of developing odontoma as a new entity arising from calcifying odontogenic cyst. Although the last WHO classification of odontogenic tumors considered the presence of dental hard tissue structures within an ameloblastic fibroma-like tissue as early stage of a developing odontoma, there are still some argue regarding whether all AFO represent early stage of odontoma. Soluk Tekkesin and Verde suggested that a combination of a cut-off age over 13.5 and size bellow $2.1 \mathrm{~cm}$ can suggest a developing odontoma. Also the presence of ameloblastic fibroma-like tissue in the periphery of the lesion, lobular arrangement of stromal component in a way that hypercellular areas being located mostly around the epithelial component, a well-developed ameloblastic epithelium and also the presence of ghost cells and cystic structures can serve as histological clues that helps to differentiate developing odontoma from AFO. ${ }^{9}$ Except to histopathologic findings, this concept also revealed that our case represents a developing odontoma.

Developing odontoma is a rare odontogenic tumor. It shows proliferation of odontogenic epithelium and primitive ectomesenchymal tissue in association with tooth structure. ${ }^{2}$ It accounts for about $3 \%$ of odontogenic tumors and usually occurs in patients younger than 20 years old. ${ }^{3} 75 \%$ of developing odontomas are located in the mandible and $67 \%$ of these are located in the posterior mandible. Over $95 \%$ of developing odontoma are associated with impacted permanent teeth and present clinically as painless slow-growing mass. Radiographically, developing odontoma depicts a clearly defined mixed unilocular or multilocular lesion with various amounts of radiopaque calcifications. ${ }^{10}$

$\mathrm{COC}$ is a rare odontogenic lesion presented as a painless slow-growing lesion with predilection for the anterior region of the jaws. It affects both maxilla and mandible equally. ${ }^{11}$ It also occurs equally in males and females and shows no race predilection. Intraosseous COC lesions are more common than the peripheral forms. ${ }^{2}$ COC affects patients between 5 to 92 years and the age of peak incidence is between the second and sixth decade of life. ${ }^{12}$ Radiographically, it shows either well-defined unilocular or multilocular radiolucencies and sometimes diffuse radiopacities. ${ }^{11}$

COC may occur in association with an impacted tooth. The distinguishing histopathologic feature of COC is the presence of ghost cells, which may calcify, in an ameloblast-like epithelium. ${ }^{11}$ It can arise in association with other odontogenic lesions like odontoma, ameloblastic fibroma, and ameloblastic fibro-odontoma (AFO). The most frequent concurrent odontogenic lesion with COC is odontoma. ${ }^{6}$ Association of COC with ameloblastic fibro-odontoma is extremely rare and so far, only 3 cases have been reported. ${ }^{8,13,14}$

The first case of Calcifying odontogenic cyst with ameloblastic fibro-odontoma was reported by Farman, et al in $1978 .^{8}$

Matsuzaka, et al reported a case of ameloblastic fibro-odontoma arising from a calcifying odontogenic cyst in 
2001. The patient was a 23 year-old male with the chief complaint of painful swelling on the left mandibular molar region. It was a multilocular mixed lesion in the panoramic radiograph. Tooth impaction was also evident. ${ }^{13}$

Lee et al., in 2014 reported calcifying odontogenic cyst associated with ameloblastic fibro-odontoma of the anterior mandible in a 4 old-year girl. The chief complaint of the lesion was swelling. It had caused tooth displacement. It was a unilocular mixed lesion that caused root resorption and cortical perforation. The lesion was also around an impacted tooth. ${ }^{14}$

Except for these two combinations of COC with ameloblastic fibro-odontoma, Imani, et al in 2017 also reported a hybrid odontogenic tumor in a 14 old year boy with a painless lesion in the left maxillary canine which was without expansion. The lesion had a mixed radiolucent-radiopaque appearance with a well-defined border in the panoramic radiograph. In histopathologic examination combination of three odontogenic lesions including calcifying odontogenic cyst, complex odontoma and ameloblastic fibro-odontoma were evident. ${ }^{15}$

The mechanism that causes to arise two odontogenic lesions together is not well known. Nevertheless, various theories have been proposed to explain the phenomenon including a transformation of one lesion into another, a collision of two separate lesions, and an inductive effect of one lesion on the another one. ${ }^{16}$ According to histopathologic findings, the first and third theories seem to be more plausible in our case.

\section{Conclusion}

We report a unique case of developing odontoma as a new entity arising from calcifying odontogenic cyst. This report highlights the importance of accurate diagnosis that influence the treatment plan.

\section{Conflict of interest}

The authors declare no conflict of interest.

\section{Authors contribution}

AA: Gathered data from the literature and wrote the first draft of the manuscript.

MMKh: Supervised the whole process of manuscript writing and also prepared the images for the manuscript.

MMKh and NM: Performed the pathologic diagnosis.

NM: approved the final manuscript

\section{Ethical approval:}

Written informed consent was obtained from the patient and her parents.

\section{ORCID:}

Monir Moradzadeh Khiavi https://orcid.org/0000-0003-4170-9596

Nazanin Mahdavi https://orcid.org/0000-0002-0691-1580

Asoma Awudu https://orcid.org/0000-0001-8036-5031

\section{Figure legends}

Figure1. Panoramic radiograph of the lesion that shows well-defined radiolucent lesion contained areas of radiopacity around the crown of an unerupted third molar (A). Cone beam computed tomography (CBCT) view of the lesion $(\mathrm{B})$.

Figure 2. Histopathologic feature of the epithelial lining of COC with prominent ghost cells $(\times 40)(\mathrm{A})$. Sections of tooth buds in the epithelium and also in the cyst wall $(\times 100)(B)$. 
Figure 3. Sections of numerous tooth buds in different stages. Dental papilla (DP); Stellate reticulum (SR); Stratum Intermedium (SI); Outer enamel epithelium (OEE); Ameloblasts (A); Odontoblasts (Od); Enamel (E); Tubular dentin (D) $(\times 200)$

Figure 4. One -year postoperative radiograph

\section{REFERENCES}

1. Speight PM, Takata TJVA. New tumour entities in the 4th edition of the World Health Organization Classification of Head and Neck tumours: odontogenic and maxillofacial bone tumours. 2018;472(3):331-9.

2. El-Naggar AK, Chan JK, Grandis JR, Takata T, Slootweg PJ. WHO classification of head and neck tumours: International Agency for Research on Cancer (IARC); 2017.

3. 3. Buchner A, Kaffe I, Vered MJH, pathology n. Clinical and radiological profile of ameloblastic fibroodontoma: an update on an uncommon odontogenic tumor based on a critical analysis of 114 cases. Head Neck Pathol 2013;7(1):54-63.

4. Hogge M, Velez I, Kaltman S, Movahed R, Yeh F. Ghost cell odontogenic tumor associated with odontomareport of two rare cases. J Clin Pediatr Dent. 2012;36(4): 373-6.

5. Gorlin RJ, Pindborg JJ, Clausen FP, Vickers RAJOS, Oral Medicine, Oral Pathology. The calcifying odontogenic cyst-a possible analogue of the cutaneous calcifying epithelioma of Malherbe: an analysis of fifteen cases. 1962;15(10):1235-43.

6. Utumi ER, Pedron IG, da Silva LP, Machado GG and Rocha AC: Different manifestations of calcifying cystic odontogenic tumor. Einstein (Sao Paulo) 2012;10(3): 366-370 (In English, Portuguese).

7. Soluk-Tekkeşin M, Wright JMJTPD. The World Health Organization classification of odontogenic lesions: a summary of the changes of the 2017 (4th) edition. 2018;34(1):1-18.

8. Farman AG, Smith SN, Nortjé CJ, Grotepass FW. Calcifying odontogenic cyst with ameloblastic fibroodontome: one lesion or two? Journal of oral pathology. 1978;7(1):19-27.

9. Soluk-Tekkesin, M., Vered, M. Ameloblastic Fibro-Odontoma: At the Crossroad Between "Developing Odontoma" and True Odontogenic Tumour. Head and Neck Pathol 2021.

10. Watanabe M, Wakoh M, Nakajima K, Yoshida S, Sato H, Koyachi M, et al. Developing odontoma with an atypical radiological appearance: A case report. 2020;6(1):100138.

11. Reyes D, Villanueva J, Espinosa S, Cornejo M. Odontogenic calcificant cystic tumor: a report of two clinical cases. Medicina oral, patologia oral y cirugia bucal. 2007;12(2):E126-9.

12. Kler S, Palaskar S, Shetty VP, Bhushan AJJoo, Intraosseous calcifying cystic odontogenic tumor. J oral maxillofaci path 2009;13(1):27.

13. Matsuzaka K, Inoue T, Nashimoto M, et al. A case of an ameloblastic fibro-odontoma arising from a calcifying odontogenic cyst. The Bulletin of Tokyo Dental College. 2001;42(1):51-5

14. Lee J, Song Y-G, Moon S-Y, Choi B, Kim BC, Yoon J-HJJoCS. Calcifying cystic odontogenic tumor associated with ameloblastic fibro-odontoma of the anterior mandible. 2014;25(3):e259-e60.

15. Imani R, Jamaatloo N, Seyedmajidi M, Khakbaz Baboli OJJoDM, Techniques. Hybrid Odontogenic Lesion: A Rare Entity. 2017;6(1):40-3.

16. Mahdavi N, Khoozestani NK, Hasheminasab M, Soltani NJJoD. Hybrid Odontogenic Tumor of Calcifying Odontogenic Cyst and Ameloblastic Fibroma: a Case Report and Review of Literature. 2020;21(2):153. 


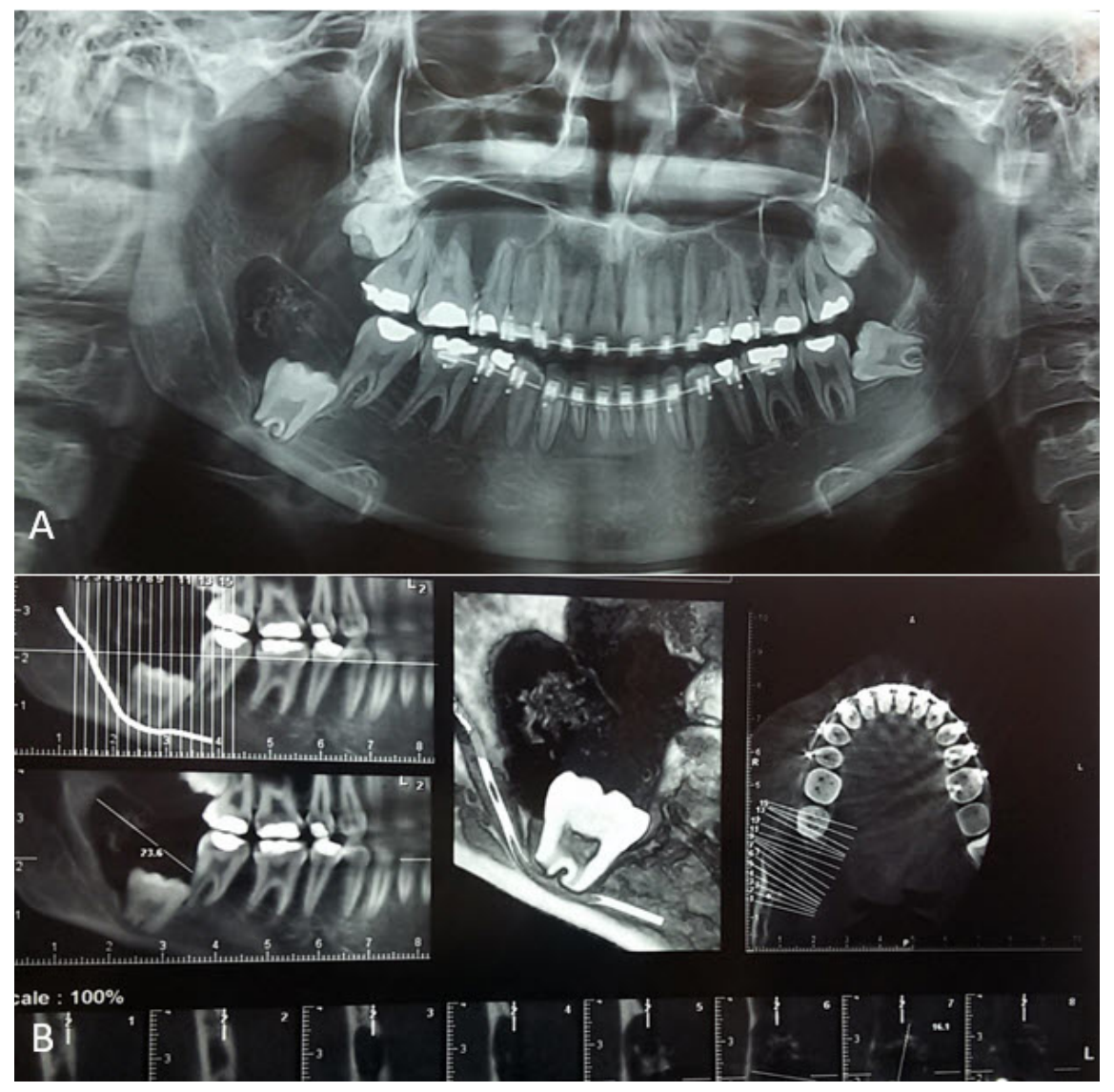



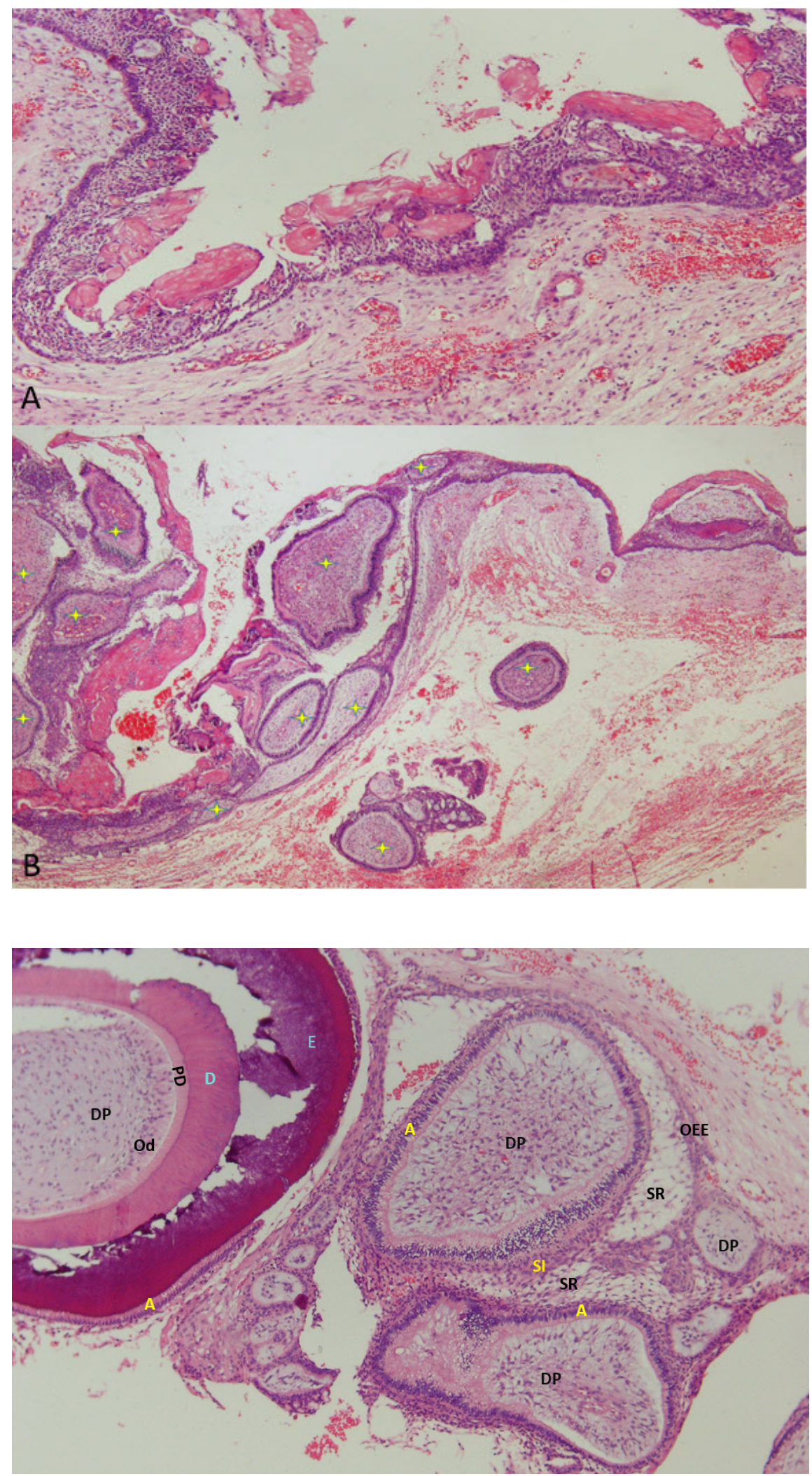


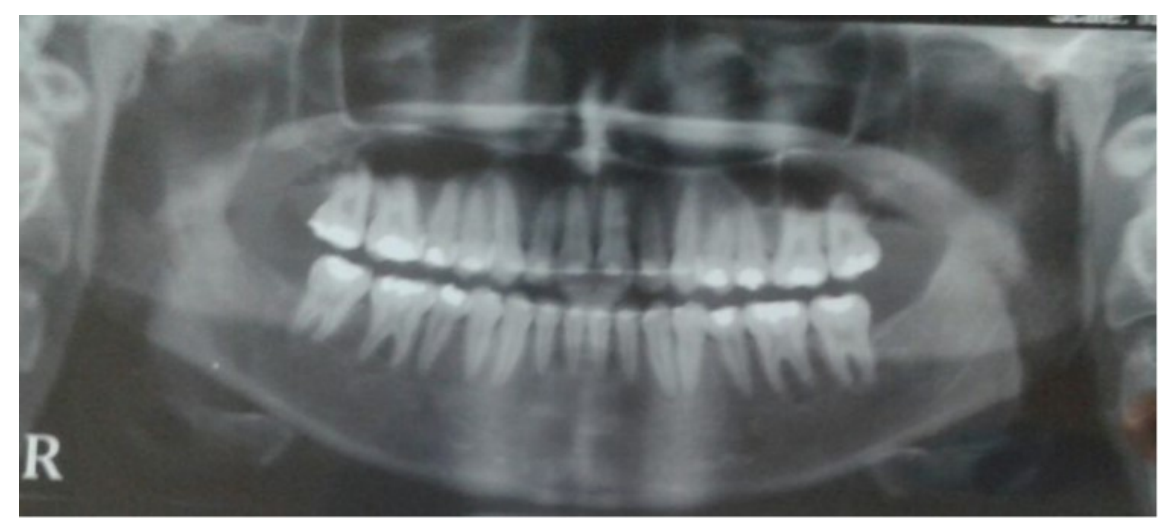

